\title{
Stratification hydrogéochimique et écoulements verticaux dans l'aquifère des calcaires de Beauce (France) : un système anthropisé à forte variabilité spatiale et temporelle
}

\author{
Hydrogeochemical stratification and vertical flows in the Beauce calcareous \\ aquifer (France): a buman-influenced system with strong spatial and temporal \\ variability
}

Nathalie Schnebelen ${ }^{\mathrm{a}, \mathrm{b}}$, Emmanuel Ledoux $^{\mathrm{b}}$, Ary Bruand $^{\mathrm{a}}$, Gilles Creuzot ${ }^{\mathrm{c}}$

a Unité de sciences du sol-SESCPF, Inra, av. de la Pomme-de-Pin, BP 20619, 45160 Olivet cedex, France

${ }^{b}$ Centre d'informatique géologique, ENSMP, 35 rue Saint-Honoré, 77305 Fontainebleau, France

c Service de l'eau et des milieux aquatiques, Diren Centre, 5 av. Buffon, BP 6407, 45064 La Source, France

(Reçu le 15 juillet 1999, accepté le 19 juillet 1999)

\begin{abstract}
Vertical profiles of temperatures and flow velocities, as well as water samples taken at different depths, reveal a hydrogeochemical stratification of the Beauce calcareous aquifer. This stratification, which is particularly well marked for the $\mathrm{NO}_{3}{ }^{-}$and $\mathrm{Cl}^{-}$profiles, is related to the development of intensive agriculture. It presents a strong variability: (i) in space because of the aquifer's multi-layered structure and the presence of reducing lcvels, (ii) and in time due to the existence in the boreholes of a vertical flow, the direction of which depends on the periodic exploitation of the aquifer. (ㅇ 1999 Académie des sciences/Éditions scientifiques et médicales Elsevier SAS.)
\end{abstract}

multi-layered aquifer / hydrogeochemical stratification / vertical flows / agriculture / France

Résumé - Dess profils verticaux de températures et de vitesses d'écoulement, ainsi que des prélèvements d'eau réalisés à différentes profondeurs, révèlent une stratification hydrogéochimique de l'aquifère des calcaires de Beauce. Cette stratification, particulièrement nette pour les profils de $\mathrm{NO}_{3}^{-}$et de $\mathrm{Cl}^{-}$, est à relier au développement d'une agriculture intensive. Elle présente une forte variabilité : (i) spatiale, liée à la structure multicouche de l'aquifère et à l'existence de niveaux réducteurs ; (ii) temporelle, due à l'existence dans les forages d'un écoulement vertical, dont le sens est lié à l'exploitation périodique de l'aquifère. (C Académie des sciences / Elsevier, Paris.)

aquifère multicouche / stratification hydrogéochimique / mouvements verticaux / agriculture / France

Note présentée par Georges Pédro.

* Correspondance et tirés à part.

Nathalie.Schnebelen@orléans.inra.fr 


\section{Abridged version}

For the last five decades human activities have been at the origin of a significant evolution of the quality of both surface and ground water (Chéverry, 1998). We already know that aquifer geochemistry can vary vertically without any lithological variation or perturbation of human origin (Pulido-Bosch et al., 1998). The airn of this study is to show that when the lithology varies and when the aquifer is affected by human activities, the aquifer geochemistry is then highly variable.

The Beauce aquifer system (figure 1) is located in the southwest of the Paris Basin and covers about $9000 \mathrm{~km}^{2}$. It develops in lacustrian facies, with calcareous dominance, which formed from the Eocene to the Aquitanian (figure 2a). The lacustrian series, which reaches $190 \mathrm{~m}$ in thickness, lies on a substratum made up of an Eocene detritic formation and on a formation made of clay and flints. Piezometric maps show the existence of two main sub-catchments (Desprez and Mégnien, 1975; BRGM, 1995): the Seine sub-catchment in the northeast of the aquifer and the Loire sub-catchment in the southwest. The main characteristic of the aquifer is the very small regional hydraulic gradient (about $0.1 \%$ ).

The site of Villamblain (figure 1) has a surface area of $7 \mathrm{~km}^{2}$ and is located in the southwest of the aquifer, in the western part of the Loire sub-catchment area. In this study area the formation, which is made of clays and flints and which constitutes the aquifer substratum, is between 15 and $25 \mathrm{~m}$ thick and the lacustrian series is about $60 \mathrm{~m}$ thick (Schnebelen et al., 1996). Upwards, the series is made up of four lithostratigraphic formations (figure $2 b$ ):

- the marly calcareous of the lacustrian Lutetian (Morancez. Limestone);

- the limestone of the lacustrian Upper Stampian (Étampes Limestone);

- the sandy marls of the Lower Aquitanian (Gâtinais Molasse); - the fissured limestone of the Upper Aquitanian (Pithiviers Calcareous).

The soils are shallow (King et al., 1999) and highly permeable (Ould Mohamed et al., 1997). The aquifer is free and the water table is located between 12 and $20 \mathrm{~m}$ depth. The transmisivity ranges from $5 \cdot 10^{-2}$ to $10^{-1} \mathrm{mi}^{2} \cdot \mathrm{s}^{-1}$ and the storage coefficient ranges from 7 to $13 \%$ (Gigot, 1973). The mean recharge is about $110 \mathrm{~mm}$ per year. Many pumps are present on the study area and are used for the irrigation of spring and summer crops.

Thermal and flow velocity profiles as well as water samples taken at different depths were carried out at different periods in eight agricultural boreholes. The thermal profiles allow location of water entering or leaving the boreholes (Jouanna, 1993). The flow velocity profiles enable the measurement of the downward and upward flow velocities (Xiang, 1995). The borcholes catch the whole aquifer thickness and allow d detailed study of the water quality variation with depth. The results presented concern the two boreholes F1 and F2, which are representative of the vertical and lateral variations observed in the aquifer beneath the study area.

During the irrigation period, the different profiles which were obtained in borehole F1 (figure 3) reveal an important hydrogeochemical stratification of the aquifer: the upper part of the aquifer (Pithiviers Limestone) presents $\mathrm{NO}_{3}^{-}$and $\mathrm{Cl}^{-}$ concentrations which are roughly greater than those recorded in the lower part (Étampes Limestone and Morancez Limestone). This hydrogeochemical stratification also exists, but is less pronounced, for other chemical clements such as $\mathrm{Na}^{+}, \mathrm{K}^{+}$ and $\mathrm{Ca}^{2+}$. The results also show a significant decrease in the $\mathrm{NO}_{3}^{-}$and $\mathrm{Cl}^{-}$concentrations passing over the sandy marls of the Gâtinais Molasse which thus constitute a partial hydraulic barrier.

Outwith the irrigation period, when the borehole is at rest, temperatures and flow velocity profiles, as well as the tracer tests and water samples taken at different depths, show the existence of an upward flow in the borehole. The lower part of the aquifer, which has an hydraulic head greater than that of the upper part, discharges into the upper part. This flow leads to an homogenisation of the chemical element concentrations (especially for $\mathrm{NO}_{3}^{-}$and $\mathrm{Cl}^{-}$) at the value of the deep horizon.

The hydrogeochemical stratification was also observed during the irrigation period in the borehole F2 (figure $\hat{4}$ ) which is locatcd at $2.6 \mathrm{~km}$ from the borehole $\Gamma 1$. I Iowever, the $\mathrm{NO}_{3}^{-}$and $\mathrm{Cl}^{-}$concentrations are much lower than those of borehole $\mathrm{F} 1$. These low concentrations are probably due to geological anomaly (marly facies of the Étampes Calcareous) and $\mathrm{NO}_{3}^{-}$ reduction processes at the bottom of the aquifer (table II). As for the borehole F1, an upward flow exists out off the irrigation period in the second borehole. However, it is not the Étampes Calcareous which present the highest hydraulic head here but underground water located in the deepest horizon.

In conclusion, our results show a significant hydrogeochemical stratification of the Beauce calcareous aquifer and both a spatial and temporal variability of this stratification. The stratification is partly explained by the structure and lithology of the aquifer, notably by the presence of an upper part which is free, of a semi-permeable layer and finally a semi-confined lower part. The hydrogeochemical composition of the upper part depends mainly on the composition of clownward flows which are affected by agriculture especially with regard to the $\mathrm{NO}_{3}^{-}$and $\mathrm{Cl}^{-}$anions. The presence of reducing levels would reduce the concentration of $\mathrm{NO}_{3}^{-}$with depth. However, the exploitation of this multi-layered aquifer induces vertical hydraulic head differences and hence rapid flows in the boreholes. These flows vary in time according to the production regime of the boreholes and can be at the origin of a gradient inversion for the $\mathrm{NO}_{3}^{-}$and $\mathrm{Cl}^{-}$anions. Similar observations have been already made by Avci (1994) and Lacombe et al. (1995). 


\section{Introduction}

Depuis une cinquantaine d'années, les activités humaines sont à l'origine d'une importante évolution de la qualité des eaux superficielles et souterraines (Chéverry, 1998). C'est en particulier le cas des aquifères libres situés dans les régions d'agriculture intensive. On sait, par ailleurs, que la géochimie d'un aquifère peut varier significativement selon la profondeur, même en l'absence de variation lithologique et de perturbation d'origine humaine (Pulido-Bosch et al., 1998).

L'objectif de cette note est de montrer que, lorsque la lithologie varie et que l'aquifère est influencé par les activités humaines, la géochimie de l'aquifère est encore plus variable. Des données acquises récemment sur le système aquifère des calcaires de Beauce, dont la lithologie est hétérogène et qui se situe dans une région d'agriculture intensive, permettent de montrer à quel point la géochimie des eaux peut varier dans l'espace, mais aussi dans le temps. On s'attachera à discuter comment de telles variations peuvent être expliquées en fonction des caractéristiques naturelles de l'aquifère et de la nature des perturbations introduites par I'homme.

\section{L'aquifère des calcaires de Beauce}

Le système aquifère de Beauce (figure 1) est situé au sud-ouest du Bassin parisien et couvre environ $9000 \mathrm{~km}^{2}$. II se développe dans un ensemble de formations lacustres à dominante calcaire qui se sont déposées durant le Tertiaire (figure 2a), depuis l'Éocène (calcaires de Morancez) jusqu'à l'Aquitanien (calcaires de Pithiviers). Le substratum de l'aquifère est constitué par des formations détritiques du Sparnacien et la formation dite à Argiles à silex, qui recouvrent la craie du Séno-Turonien. La série lacus-

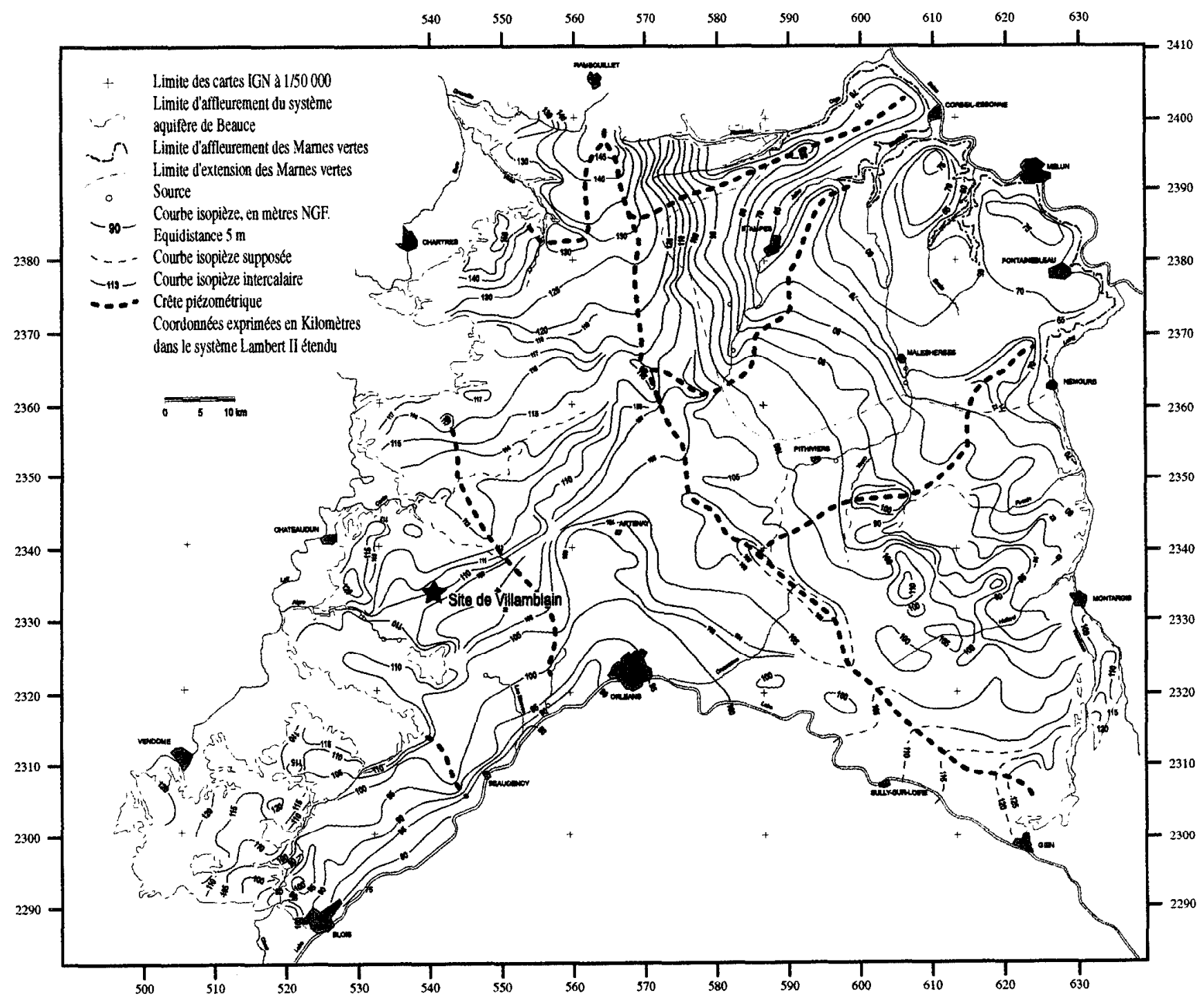

Figure 1, Délimitation du système aquifère de Beauce, piézométrie en 1994 (basses eaux) et localisation du site d'étude de Villamblain (d'après BRGM, 1995).

Delimitation of the Beauce aquifer system, water table in 1994 (low levels) and location of the Villamblain study area (after BRGM, 1995). 
tre, qui peut atteindre $190 \mathrm{~m}$ de puissance, suit les ondulations NW-SE (direction armoricaine) du substratum. Les cartes piézométriques mettent en évidence l'existence de deux principaux bassins versants souterrains (Desprez et Mégnien, 1975 ; BRGM, 1995) : le bassin de la Seine, au nord-est de l'aquifère, et le bassin de la Loire, au sudouest. Les directions d'écoulement sont respectivement SW-N[ et NW-SE, avec un très faible gradient hydraulique régional (de l'ordre de $0,1 \%$ ).

\section{Le site étudié}

Le site de Villamblain (figure 1), d'une superficie de $7 \mathrm{~km}^{2}$, est situé au sud-ouest de l'aquifère, dans la partie occidentale du bassin versant souterrain de la Loire. L'épaisseur des argiles à silex qui constituent le substratum de l'aquifère est comprise entre 15 et $25 \mathrm{~m}$; celle de la série lacustre est voisine de $60 \mathrm{~m}$ (Schnebelen et al., 1996). La série lacustre (figure $2 b$ ) débute, soit par les calcaires marneux du Lutétien (calcaires de Moranccz), qui peuvent atteindre une dizaine de mètres et qui supportent 5 à $30 \mathrm{~m}$ de calcaires du Stampien supérieur (calcaires d'Étampes), soit directement par les calcaires du Stampien. Viennent ensuite des couches de marnes sableuses attribuées à la molasse du Gâtinais (Aquitanien inférieur), dont l'épaisseur est voisine de $5 \mathrm{~m}$. Sur la zone d'étude, la série lacustre se termine par les calcaires fissurés de l'Aquitanien supérieur (calcaires de Pithiviers), dont l'épaisseur est comprise entre 20 et $30 \mathrm{~m}$. Les calcaires de Pithiviers sont recouverts par une couverture pédologique peu épaisse (King et al., 1999) et très perméable (Ould Mohamed et al., 1997).

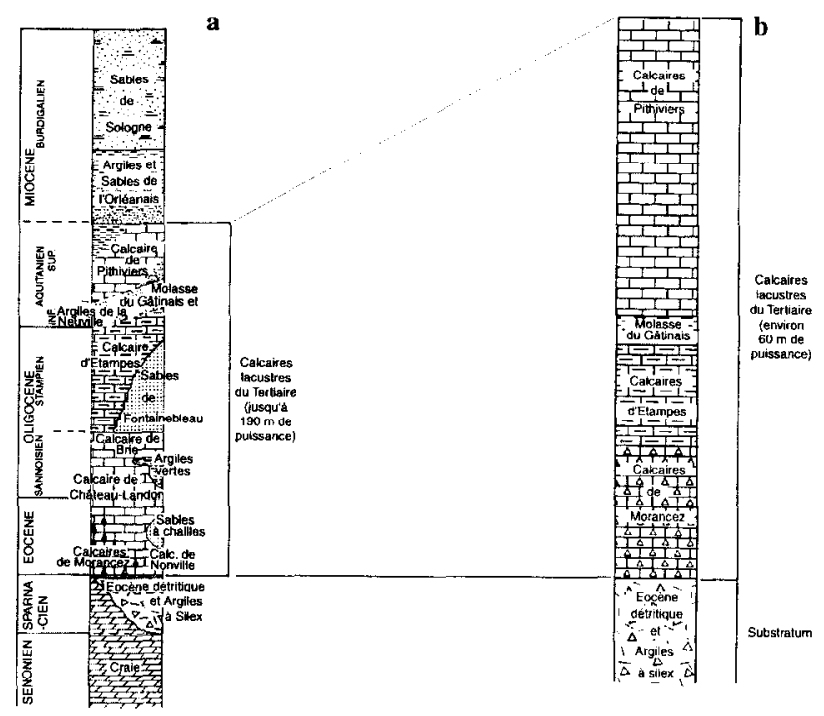

Figure 2. Colonne lithostratigraphique du système de Beauce. a : ensemble des formations (d'après Desprez et Mégnien, 1975) ; b : formations présentes au droit du site d'étude.

Lithostratigraphic column of the Beauce system. a: whole formations (after Desprez and Mégnien, 1975); b: formations present benedth the study area.
La nappe est libre et le niveau piézométrique est situé entre 12 et $20 \mathrm{~m}$ de profondeur. La transmissivité est de $5.10^{-2}$ à $10^{-1} \mathrm{~m}^{2} \cdot \mathrm{s}^{-1}$ et le coefficient d'emmagasinement est compris entre 7 et $13 \%$ (Gigot, 1973). La lame d'eau infiltrée est en moyenne de $110 \mathrm{~mm}$ par an sur la période 1946-1994. De nombreux pompages sont présents sur le site d'étude et sont utilisés pour f'irrigation printanière et estivale dans des forages dont le débit instantané varie de 60 à $270 \mathrm{~m}^{3} \cdot \mathrm{h}^{-1}$.

\section{Les mesures réalisées dans les forages}

Des profils thermiques, des profils de vitesses d'écoulement et des prélèvements d'échantillons d'eau à différentes profondeurs ont été effectués à différentes périodes dans 8 forages agricoles. Ces forages captent toute la hauteur de l'aquifère et permettent ainsi une étude détaillée de l'évolution de la qualité de l'eau avec la profondeur. Nous présentons les résultats obtenus dans deux forages $\mathrm{F} 1(X=543,68 \mathrm{~km} ; Y=2335,90 \mathrm{~km})$ et $\mathrm{F} 2$ $(X=546,16 \mathrm{~km} ; Y=2335,28 \mathrm{~km})$, qui permettent de mettre en évidence un comportement typique, représentatif des variations observées verticalement et latéralement dans l'aquifère.

Les profils de températures de l'eau dans un forage permettent, par l'analyse des perturbations du gradient de température, de localiser les arrivées et les pertes d'eau en fonction de la profondeur et renseignent, par conséquent, sur les propriétés hydrodynamiques verticales de l'aquifère (Jouanna et al., 1993). Ces profils thermiques ont été réalisés à l'aide d'une sonde piézo-température dont la résolution est le centième de degré. La température a été mesurée par paliers descendants et remontants, tous les 0,5 ou $1 \mathrm{~m}$, sur toute la colonne d'eau de chaque forage.

Les profils de débits ont été réalisés à l'aide d'un micromoulinet à cellule photoélectrique. Cel appareil permel de mesurer des vitesses de courants verticaux, ascendants ou descendants, à l'intéricur d'un forage et de déterminer ainsi les zones de production ou de pertes (Xiang, 1995).

Trois campagnes de mesure ont été réalisées :

- une campagne de juin à septembre 1996, pendant la période d'irrigation estivale, lorsque les forages sont en cours de pompage ;

- deux autres campagnes, I'une à l'hiver 1996, I'autre en mars 1997 , alors que les pompages agricoles étaient arrêtés.

Après avoir localisé les arrivées d'eau à l'aide des profils thermiques et des profils de vitesses d'écoulement, des prélèvements ont été effectués en face de chaque arrivée, afin d'en déterminer les caractéristiques physicochimiques. L'analyse des espèces chimiques majeures a été effectuée par chromatographie en phase liquide. 


\section{Résultats et discussion}

\subsection{Mise en évidence d'une stratification hydrogéochimique}

Le forage $\mathrm{F} 1$ a une profondeur de $55 \mathrm{~m}$ et il est crépiné à partir de $19 \mathrm{~m}$. Il capte donc l'ensemble de la série lacustre (figure $3 a$ ), des calcaires de Pithiviers aux calcaires de Morancez inclus. Pendant la période d'irrigation, les écoulements dans le forage (figure $3 b$ ) sont descendants au-dessus de la crépine du groupe électropompe, qui est située à $30 \mathrm{~m}$ de profondeur, et ascendants audessous de celle-ci. Six arrivées d'eau, matérialisées par des zones où le débit augmente (figure $3 c$ ) et où le gradient de température varie (figure $3 b$ ), ont été mises en évidence. Ainsi, deux arrivées d'eau, notées 1 et 2 sur la figure $3 b$, ont été repérées à la hauteur des calcaires de Pithiviers, trois à la hauteur des calcaires d'Étampes (arrivées 3,4 et 5) et une à la hauteur des calcaires de Morancez (notée 6).

Les prélèvements (figures $3 d$ et 3 e) révèlent que les eaux issues des calcaires de Pithiviers possèdent, pour ce forage, des teneurs en $\mathrm{NO}_{3}^{-}$et en $\mathrm{Cl}^{-}$élevées : $79 \mathrm{mg} \cdot \mathrm{L}^{-1}$ de $\mathrm{NO}_{3}^{-}$et $35 \mathrm{mg} \cdot \mathrm{L}^{-1}$ de $\mathrm{Cl}^{-}$pour l'arrivée d'eau notée 1 et $85 \mathrm{mg} \cdot \mathrm{L}^{-1}$ de $\mathrm{NO}_{3}^{-}$el $36 \mathrm{mg} \cdot \mathrm{L}^{-1}$ de $\mathrm{Cl}^{-}$pour le mélange des arrivées d'eau 1 et 2 . En revanche, les teneurs en $\mathrm{NO}_{3}^{-}$ et en $\mathrm{Cl}^{-}$sont moins élevées pour les arrivées d'eau situées à la hauteur des calcaires d'Étampes et des calcaires de Morancez: voisines de $50 \mathrm{mg} \cdot \mathrm{L}^{-1}$ pour $\mathrm{NO}_{3}^{-}$et de $20 \mathrm{mg} \cdot \mathrm{L}^{-1}$ pour $\mathrm{Cl}^{-}$. L'échantillon prélevé à la base des calcaires de Morancez $(53 \mathrm{~m})$ présente une teneur en $\mathrm{NO}_{3}^{-}$de $39 \mathrm{mg} \cdot \mathrm{L}^{-1}$, alors que la teneur en $\mathrm{Cl}^{-}$reste in- changée. Les dosages d'autres éléments chimiques effectués sur cet échantillon (tableau I) montrent que cette eau est plus ancienne (traces de fluor) et plus réductrice (présence de nitrites), même si la teneur en $\mathrm{NO}_{3}^{-}$reste élevée. Il s'agit là sans doute d'un horizon peu productif des calcaires de Morancez.

Par conséquent, ces mesures révèlent une importante stratification hydrogéochimique : la partie supérieure de I'aquifère (calcaires de Pithiviers) possède des teneurs en $\mathrm{NO}_{3}^{-}$et en $\mathrm{Cl}^{-}$presque 2 fois supérieures à celles observées dans la partie inférieure (calcaires d'Étampes et calcaires de Morancez). Cette stratification hydrogćochimique existe aussi, mais de façon moins marquée, pour $\mathrm{d}^{\prime}$ autres éléments chimiques tels que $\mathrm{Na}^{+}, \mathrm{K}^{+}$et $\mathrm{Ca}^{2+}$ (tableau 1). Ces résultats révèlent aussi une forte diminution des teneurs en $\mathrm{NO}_{3}^{-}$et en $\mathrm{Cl}^{-}$al passage de la molasse du Gâtinais. Celle-ci constituerait en quelque sorte un écran limitant les transferts verticaux et ainsi le mélange des eaux récentes provenant de la surface avec les eaux plus anciennes qui sont présentes en profondeur.

\subsection{Présence d'écoulements verticaux}

En dehors de la période d'irrigation (décembre 1996 et mars 1997), lorsque le forage est au repos, les profils thermiques obtenus dans le forage $\mathrm{F} 1$ (figure $3 \mathrm{~b}$ ) sont, pour la partie inférieure de l'aquifère, peu différents de celui réalisé en juin 1996 ; on remarque les mêmes variations du gradient thermique, témoins d'arrivées d'eau. En revanche, pour la partie supérieure de l'aquifère, les profils thermiques sont décalés vers des températures plus élevées et présentent un aspect uniforme autour de la valeur $12,20^{\circ} \mathrm{C}$.

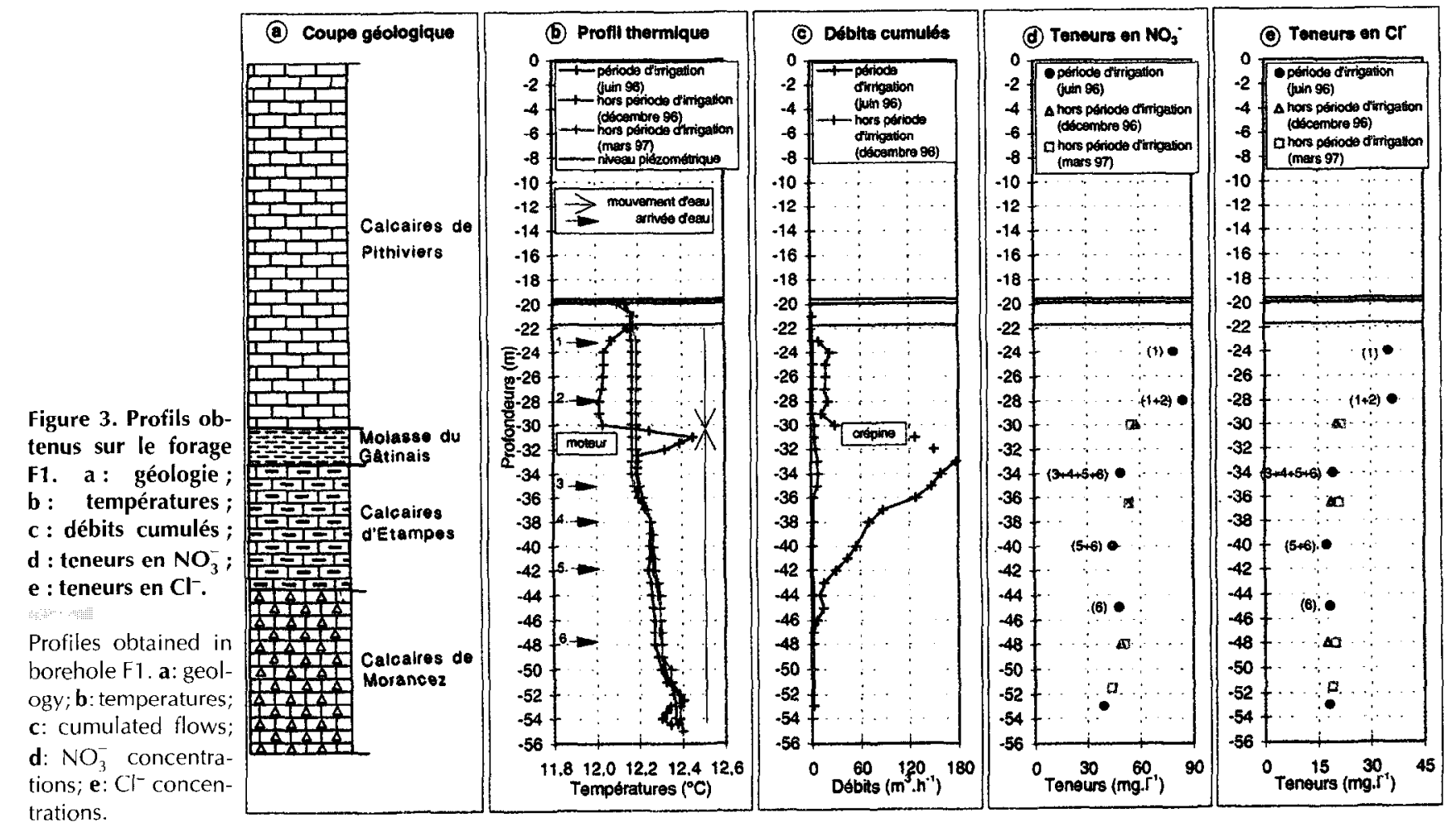

C. R. Acad. Sci. Paris, Sciences de la terre et des planètes / Earth \& Planetary Sciences 
Tableau I. Analyses chimiques des échantillons d'eaux prélevés dans le forage F1 (PI : période d'irrigation ; HPI : hors période d'irrigation ; profondeur en $\mathbf{m}$; concentrations en $\left.\mathbf{m g} \cdot \mathrm{L}^{-1}\right)$.

Chemical analyses of the water samples collected in borehole $\mathrm{F} 1$ ( $\mathrm{Pl}$ : irrigation period; HPl: outwith irrigation period; depth in m; concentrations in $\left.\mathrm{mg} \cdot \mathrm{L}^{-1}\right)$.

\begin{tabular}{|c|c|c|c|c|c|c|c|c|c|c|c|c|c|c|c|c|c|c|c|c|c|c|}
\hline & \multicolumn{2}{|c|}{ Profondeurs } & \multicolumn{20}{|c|}{ Concentrations } \\
\hline & \multirow[b]{2}{*}{$\mathrm{Pl}$} & \multirow[b]{2}{*}{$\mathrm{HPI}$} & \multicolumn{2}{|c|}{$\mathrm{Na}^{+}$} & \multicolumn{2}{|c|}{$\mathrm{K}^{+}$} & \multicolumn{2}{|c|}{$\mathrm{Mg}^{2+}$} & \multicolumn{2}{|c|}{$\mathrm{Ca}^{2+}$} & \multicolumn{2}{|c|}{$\mathrm{HCO}_{3}^{-}$} & \multicolumn{2}{|c|}{$\mathrm{F}^{-}$} & \multicolumn{2}{|c|}{$\mathrm{Cl}^{-}$} & \multicolumn{2}{|c|}{$\mathrm{NO}_{3}^{-}$} & \multicolumn{2}{|c|}{$\mathrm{NO}_{2}^{-}$} & \multicolumn{2}{|c|}{$\mathrm{SO}_{4}^{2-}$} \\
\hline & & & PI & $\mathrm{HPI}$ & PI & $\mathrm{HPI}$ & PI & $\mathrm{HPI}$ & $\mathrm{PI}$ & $\mathrm{HPI}$ & $\mathrm{Pl}$ & $\mathrm{HPI}$ & $\mathrm{PI}$ & $\mathrm{HPl}$ & $\mathrm{Pl}$ & $\mathrm{HPI}$ & PI & $\mathrm{HPI}$ & $\mathrm{Pl}$ & $\mathrm{HPI}$ & $\mathrm{Pl}$ & $\mathrm{HPI}$ \\
\hline Calcaires de & -24 & & 8,0 & & 2,9 & & 4,6 & & 106 & & 207 & & 0,2 & & 35 & & 79 & & 0,0 & & 5,5 & \\
\hline Pithiviers & -28 & & 7.6 & & 2,0 & & 4,5 & & 108 & & 210 & & 0.1 & & 36 & & 85 & & 0,0 & & 5,1 & \\
\hline $\begin{array}{l}\text { Molasse du } \\
\text { Gâtinais }\end{array}$ & & -30 & & 5,6 & & 1,5 & & 4,8 & & 89 & & 227 & & 0,0 & & 22 & & 55 & & 0,0 & & 3,8 \\
\hline Calcaires & -34 & $-4,7$ & 5,7 & 5,3 & 1,5 & 1,4 & 4,1 & 4,7 & 93 & 87 & 210 & 231 & 0,1 & 0,0 & 19 & 21 & 49 & 54 & 0,0 & 0,0 & 5,1 & 3,8 \\
\hline d'Étampes & -40 & & 5,7 & & 1,5 & & 4,0 & & 92 & & 205 & & 0,1 & & 18 & & 45 & & 0,0 & & 4,7 & \\
\hline Calcaires de & -45 & -48 & 5,4 & 5,2 & 1,4 & 1,4 & 4,1 & 5,5 & 92 & 85 & 206 & 228 & 0,1 & 0,0 & 18 & 20 & 48 & 51 & 0,0 & 0,0 & 5,2 & 5,4 \\
\hline Morancez & -53 & -52 & 6,1 & 5,1 & 1,6 & 1,1 & 7,6 & 7,2 & 89 & 81 & 216 & 237 & 0,2 & 0,3 & 18 & 19 & 39 & 44 & 0.1 & 0,0 & 11,2 & 8,5 \\
\hline
\end{tabular}

La température de $12,20^{\circ} \mathrm{C}$ mesurée à la hauteur des calcaires de Pithiviers correspond en réalité à la température de l'arrivée d'eau située à $35 \mathrm{~m}$ de profondeur, au sommet des calcaires d'Étampes (arrivée 3). Ceci s'interprète par le fait que cet horizon des calcaires d'Étampes possède une piézométrie plus élevée, provoquant ainsi une décharge des horizons producteurs du calcaire d'Étampes dans les horizons du calcaire de Pithiviers, qui deviennent absorbants. Ce résultat est corroboré par le profil de débits obtenu au micromoulinet dans le forage au repos. En effet, des vitesses voisines de $1 \mathrm{~cm} \cdot \mathrm{s}^{1}$, correspondant à un débit de $9 \mathrm{~m}^{3} \cdot \mathrm{h}^{-1}$, ont été mesurées au micromoulinet entre 35 et $29 \mathrm{~m}$ de profondeur, à la hauteur du toit des calcaires d'Étampes et de la molasse du Gâtinais. Cette valeur est confirmée par une expérience de traçage dans le forage, où l'on a observé la vitesse de déplacement vertical d'un pic d'eau salée. D'autre part. on constate que les teneurs en $\mathrm{NO}_{3}^{-}$et en $\mathrm{Cl}^{-}$des échantillons d'eau prélevés dans la partie supérieure de l'aquifère sont nettement plus faibles en dehors de la période $\mathrm{d}^{\prime}$ irrigation que pendant la période d'irrigation (figures $3 d$ et $3 e)$ : $55 \mathrm{mg} \cdot \mathrm{L}^{-1}$ contre $85 \mathrm{mg} \cdot \mathrm{L}^{-1}$ pour $\mathrm{NO}_{3}^{-}$et $22 \mathrm{mg} \cdot \mathrm{L}^{-1}$ contre $36 \mathrm{mg} \cdot \mathrm{L}^{-1}$ pour $\mathrm{Cl}^{-}$. Les teneurs en $\mathrm{NO}_{3}^{-}$ et en $\mathrm{Cl}^{-}$, voisines de $50 \mathrm{mg} \cdot \mathrm{L}^{-1}$ et de $20 \mathrm{mg} \cdot \mathrm{L}^{-1}$ respectivement, correspondent en réalité aux teneurs dans la partie inférieure de l'aquifère. Des teneurs identiques entre la partie supérieure de l'aquifère et la partie inférieure ont également été mesurées en dehors de la période d'irrigation, pour d'autres éléments chimiques (tableau I).

On a donc mis en évidence, pendant la période d'arrêt des pompages, une circulation ascendante dans le forage. La partie inférieure de l'aquifère (calcaires d'Étampes) est en charge et débite, au travers du forage, dans la partie supérieure (calcaires de Pithiviers). Ceci homogénéise les teneurs en éléments chimiques (notamment en $\mathrm{NO}_{3}^{-}$et en $\mathrm{Cl}^{-}$) à la valeur correspondant aux horizons profonds.

\subsection{Appréciation de la variabilité latérale}

Le forage $F 2$ est situé à $2,6 \mathrm{~km}$ à l'est du forage $F 1$. Sa profondeur est de $66 \mathrm{~m}$ et il est crépiné à partir de $21 \mathrm{~m}$. II capte donc (figure 4a) l'ensemble de la série lacustre, sauf en ce qui concerne les calcaires de Morancez, qui ne sont pas représentés au niveau de ce forage, et une partie du substratum constitué par de l'Éocène détritique et des Argiles à silex, avec une forte teneur en silex. Le profil thermique (figure $4 b$ ) et le profil de débits (figure $4 c$ ) réalisés pendant la période d'irrigation mettent en évidence 4 arrivées d'eau. Ces arrivées sont situées respectivement à la base des calcaires de Pithiviers (arrivée 1), dans les calcaires d'Étampes (arrivée 2) et à la hauteur de l'Éocène détritique et de la formation dite à argiles à silex (arrivées 3 et 4). Cependant, l'augmentation du débit (figure 4C) est relativement limitée à la hauteur des calcaires d'Étampes, alors qu'elle est beaucoup plus importante à la hauteur du substratum. Les calcaires d'Étampes, connus pour être très productifs (Desprez et Mégnien, 1975), ne le sont pas au niveau de ce forage, car ils sont très marneux, comme le montre la description de la coupe géologique locale. En revanche, la formation du substratum est à l'origine d'une importante arrivée d'eau. Cette dernière serait liée au faciès très caillouteux (teneur élevée en silex) de la formation à cet endroit.

Pour ce qui concerne l'arrivée d'eau issue des calcaires de Pithiviers, les leneurs mesurées pendant la période $\mathrm{d}^{\prime}$ irrigation sont voisines, respectivement, de $30 \mathrm{mg} \cdot \mathrm{L}^{-1}$ pour $\mathrm{NO}_{3}^{-}$(figure $4 d$ ) et de $15 \mathrm{mg} \cdot \mathrm{L}^{-1}$ pour $\mathrm{Cl}^{-}$(figure 4e). En revanche, pour les arrivées d'eau issues de l'Éocène détritique et de la formation dite à argiles à silex, la teneur en $\mathrm{NO}_{3}^{-}$est inférieure au seuil de détection 


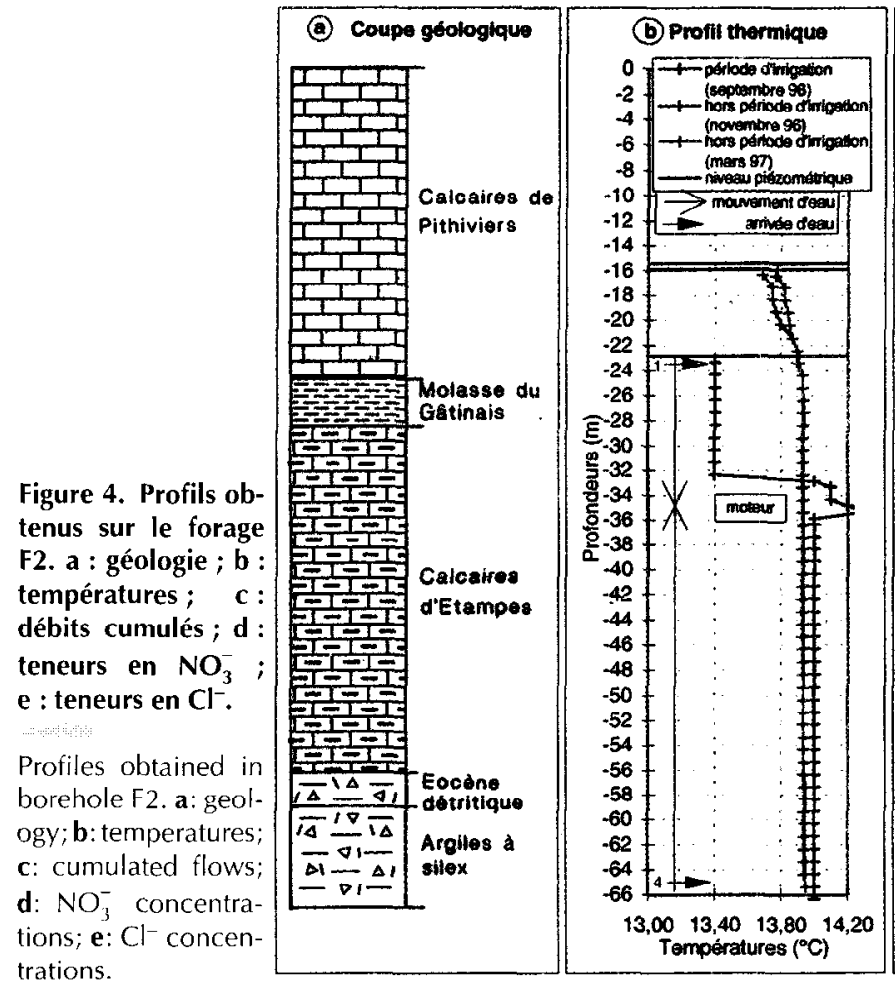

$\left(5 \cdot 10^{-3} \mathrm{mg} \cdot \mathrm{L}^{-1}\right)$ et la teneur en $\mathrm{Cl}^{-}$est de $7 \mathrm{mg} \cdot \mathrm{L}^{-1}$. Cette diminution des teneurs en $\mathrm{NO}_{3}^{-}$s'accompagne d'une baisse des valeurs en oxygène dissous et du potentiel redox, ainsi que d'une augmentation des teneurs en sulfates (tableau 1 ). Ces évolutions sont généralement associées à un processus de réduction naturelle de $\mathrm{NO}_{3}^{-}$sur substrats soufrés (Edmunds et Walton, 1983), qui pourraient provenir de la pyrite contenue dans certains dépôts de l'Éocène détritique.

Ainsi, la stratification hydrogéochimique de l'aquifère a également été observée pendant la période d'irrigation au niveau de ce second forage. Cependant, les tencurs en $\mathrm{NO}_{3}^{-}$et en $\mathrm{Cl}^{-}$sont nettement plus faibles que pour le forage $\mathrm{F} 1$.

En dehors de la période d'irrigation (novembre 1996 et mars 1997), les profils thermiques (figure 4 b) sont uniformes sur toute la hauteur crépinée du forage, soit de 21 à $66 \mathrm{~m}$ de profondeur. La température de $13,94{ }^{\circ} \mathrm{C}$ correspond à la température de l'arrivée d'eau issue de la formation basale (arrivée 4). Les 3 autres arrivées d'eau, mises en évidence, pendant la période d'irrigation, à la hauteur de l'Éocène détritique, des calcaires d'Étampes et des calcaires de Pithiviers, n'apparaissent plus sur les profils thermiques. L'horizon producteur de la formation basale est donc en charge et débite dans les couches supérieures. Les teneurs en $\mathrm{NO}_{3}^{-}$et en $\mathrm{Cl}^{-}$mesurées en dehors de la période d'irrigation (figures $4 d$ et $4 e$ ) sont cohérentes avec cette interprétation: toute la colonne d'eau du forage possède les mêmes teneurs en $\mathrm{NO}_{3}^{-}$et en $\mathrm{Cl}^{-}$que l'arrivée d'eau de la formation basale, soit inférieure à $5 \cdot 10^{-3} \mathrm{mg} \cdot \mathrm{L}^{-1}$ pour $\mathrm{NO}_{3}^{-}$et environ $7 \mathrm{mg} \cdot \mathrm{L}^{-1}$ pour $\mathrm{Cl}^{-}$. Il en est de même pour les autres éléments chimiques
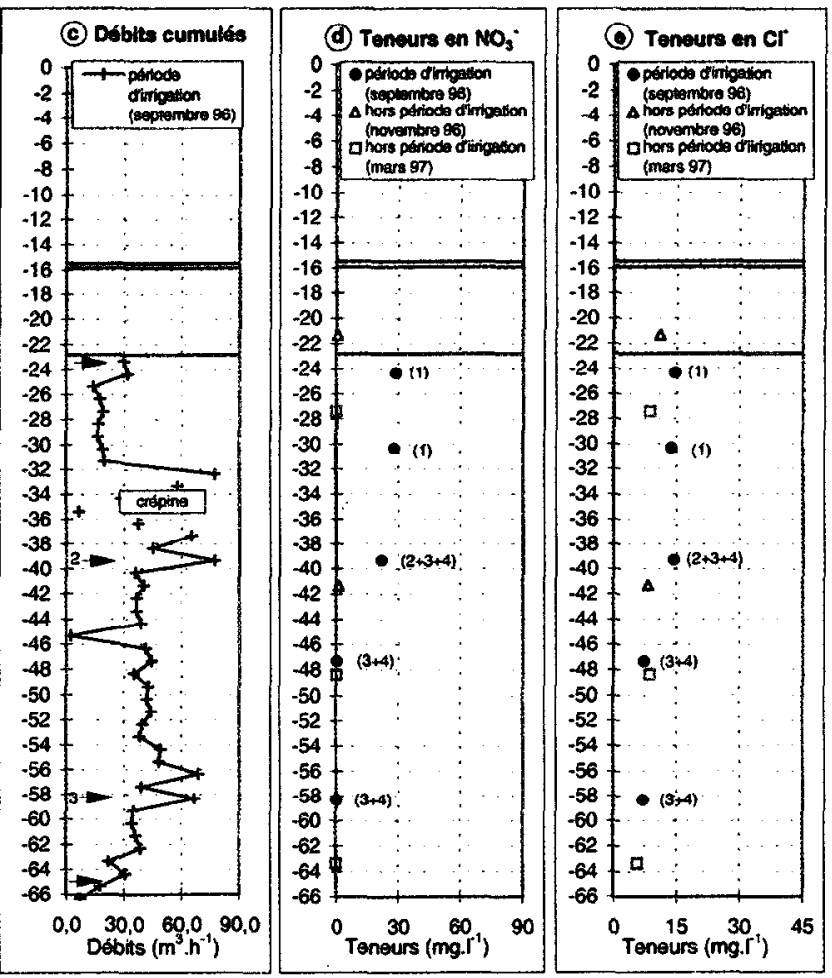

analysés, à l'exception des sulfates (tableau In. Un profil au micromoulinet a été réalisé en dehors de la période d'irrigation, mais il n'est pas exploitable, la vitesse de déplacement étant trop faible (inférieure au seuil de rotation de l'hélice, soit $0,4 \mathrm{~cm} \cdot \mathrm{s}^{-1}$ ). Une expérience de traçage donne, en revanche, un débit de percolation verticale de $9 \mathrm{~m}^{3} \cdot \mathrm{h}^{-1}$.

Comme pour le forage $\mathrm{F} 1$, une circulation locale ascendante existe, en dehors de la période d'irrigation, dans ce second forage. Toutefois, ce ne sont pas les calcaires d'Étampes qui présentent ici la charge la plus élevée, mais une nappe localisée dans un horizon plus profond.

\section{Conclusion}

Lcs résultats présentés dans cette note révèlent une importante stratification hydrogéochimique de l'aquifère des calcaires de Beauce, ainsi qu'une forte variabilité spatiale et temporelle de cette stratification.

La stratification hydrogéochimique est en premier lieu expliquée par la structure et la lithologie des formations géologiques dans lesquelles l'aquifère se développe. Elle est particulièrement nette pour les aquifères qui comportent, comme l'aquifère des calcaires de Beauce, une partie supérieure libre, un niveau semi-perméable et une partie inférieure semi-captive.

Dans de tels aquifères, la composition hydrogéochimique de la partie supérieure libre dépend directement de la composition des écoulements verticaux per descensum, qui participent à la recharge de l'aquifère et dont la composition est largement influencée par les activités agricoles. C'est notamment le cas des anions $\mathrm{NO}_{3}^{-}$et $\mathrm{Cl}^{-}$. 
Tableau II. Analyses chimiques des échantillons d'eaux prélevés dans le forage $\mathrm{F} 2$ ( $P I$ : période d'irrigation ; $\mathrm{HPI}$ : hors période d'irrigation ; profondeur en $\mathrm{m}$; concentrations en $\mathrm{mg} \cdot \mathrm{L}^{-1}$ ).

Chemical analyses of the water samples collected in the borehole F) (Pl: irrigation period; HPl: outwith irrigation period; depth in m; concentrations in $\left.\mathrm{mg} \cdot \mathrm{L}^{-1}\right)$.

\begin{tabular}{|c|c|c|c|c|c|c|c|c|c|c|c|c|c|c|c|c|c|c|c|c|c|c|}
\hline & \multicolumn{2}{|c|}{ Profondeurs } & \multicolumn{20}{|c|}{ Concentrations } \\
\hline & \multirow[b]{2}{*}{$\mathrm{Pl}$} & \multirow[b]{2}{*}{$\mathrm{HPI}$} & \multicolumn{2}{|c|}{$\mathrm{Na}^{+}$} & \multicolumn{2}{|c|}{$\mathrm{k}^{+}$} & \multicolumn{2}{|c|}{$\mathrm{Mg}^{2+}$} & \multicolumn{2}{|c|}{$\mathrm{Ca}^{2+}$} & \multicolumn{2}{|c|}{$1 \mathrm{ICO}_{3}^{-}$} & \multicolumn{2}{|c|}{$\mathrm{F}^{+}$} & \multicolumn{2}{|c|}{$\mathrm{Cl}^{+}$} & \multicolumn{2}{|c|}{$\mathrm{NO}_{3}^{-}$} & \multicolumn{2}{|c|}{$\mathrm{NO}_{2}^{-}$} & \multicolumn{2}{|c|}{$\mathrm{SO}_{4}^{2-}$} \\
\hline & & & $\mathrm{PI}$ & $\mathrm{HPI}$ & $\mathrm{Pl}$ & $\mathrm{HPI}$ & $\mathrm{PI}$ & $\mathrm{HPI}$ & $\mathrm{Pl}$ & $\mathrm{HPI}$ & $\mathrm{Pl}$ & HPI & $\mathrm{PI}$ & $\mathrm{HPI}$ & $\mathrm{Pl}$ & $\mathrm{HPI}$ & $\mathrm{Pl}$ & $\mathrm{HPI}$ & PI & $\mathrm{HPI}$ & $\mathrm{Pl}$ & $\mathrm{HPl}$ \\
\hline $\begin{array}{l}\text { Molasse du } \\
\text { Gâtinais }\end{array}$ & -24 & -27 & 6,8 & 4,7 & 1,8 & 1,5 & 10,5 & 12,6 & 90 & 72 & 228 & 254 & 0,2 & 0,4 & 14,7 & 8,7 & 29 & 0 & 0,0 & 0,0 & 17 & 33 \\
\hline \multirow{3}{*}{$\begin{array}{l}\text { Calcaires } \\
\text { d'Étampes }\end{array}$} & -30 & & 6,7 & & 1,9 & & 10,7 & & 87 & & 249 & & 0,3 & & 13,8 & & 28 & & 0,0 & & 17 & \\
\hline & -39 & & 6,0 & & 2,0 & & 14,7 & & 77 & & 245 & & 0,1 & & 14,3 & & 22 & & 0,0 & & 23 & \\
\hline & -47 & -48 & 5,7 & 4,7 & 1,7 & 1,5 & 13,1 & 12,6 & 79 & 71 & 245 & 254 & 0,4 & 0,4 & 7,3 & 8,7 & 0 & 0 & 0,0 & 0,0 & 30 & 33 \\
\hline Argiles à silex & 58 & 63 & 6,0 & 4,9 & 2,0 & 1,5 & 13,3 & 12,6 & 80 & 73 & 211 & 246 & 0,1 & 0,3 & 7,1 & 5,8 & 0 & 0 & 0,0 & 0,0 & 30 & 20 \\
\hline
\end{tabular}

En profondeur, des niveaux réducteurs peuvent aussi être présents localement. Ils sont alors aussi à l'origine de variations de composition hydrogéochimique, et sont en particulier responsables d'une diminution de la concentration en $\mathrm{NO}_{3}^{-}$.

Cependant, l'exploitation d'un tel aquifère multicouche induit verticalement des différences de charges hydrauliques. Il en résulte alors des écoulements rapides dans les forages, qui varient dans le temps selon le régime de production des puits et peuvent être à l'origine d'une inversion du profil de concentration des anions $\mathrm{NO}_{3}^{-}$et $\mathrm{Cl}^{-}$. En période de pompage, le mélange d'eau provenant des différents horizons producteurs conduit à des teneurs en éléments chimiques en sortie de forage qui sont les moyennes des teneurs dans les différents horizons, pondé- rées par les débits de ces horizons. Lorsque le forage est au repos, il se produit en général un écoulement ascendant qui entraîne une homogénéisation des teneurs à la valeur des horizons profonds. Des observations analogues ont été réalisées par Avci (1994) et Lacombe et al. (1995).

De manière plus générale, les résultats présentés dans cette note montrent que la composition hydrogéochimique d'une eau issue d'un système aquifère comme celui de Beauce ne peut en aucun cas être interprétée sans connaissance préalable du fonctionnement du système aquifère et de son régime de production tout au long de l'année. C'est en effet ainsi qu'il sera possible de replacer les données hydrogéochimiques dans leur contexte spatial et temporel, ainsi que d'en tirer toutes les conséquences en matière de qualité de l'eau.

Remerciements. Cette étude a été effectuée dans le cadre du contrat de rechercho "Villamblain-Modélisation", dont lo financement a été assuré par l'Agence de l'eau Loire-Bretagne et la région Centre. Nous tenons à remercier ces partenaires, ainsi que les propriétaires des forages agricoles.

\section{Références}

Avci C.B. 1994. Evaluation of flow leakage through abandoned wells and boreholes, Water Resour. Res., 9, 2565-2578

BRGM, 1995. Piézométrie du syslème aquirèe de Beauce - Basses eaux 1994, Rapport BRGM R38572, 33 p.

Chéverry C. 1998. Agriculture intensive et qualité des eaux, Éditions Inra, Versailles, $297 \mathrm{p}$.

Edmunds W.M. et Walton N.R.G. 1983. The Lincolnshire limestorle : hydrogeochemical evolution over a ten-year period. Process in karst hydrogeology. Stringfield Memorial Volume, J. Hydrol, 61, 201-211

Desprez N. et Mégnien C. 1975. Atlas hydrogéologique de la Beauce, BRGM, Service géologique régional du bassin de Paris, Orléans, $112 \mathrm{p}$.

Gigot C. 1973. Notice explicative de la carte géologique de la France au 1/50 000, feuille 362, Patay (21-19)

Jouanna P. et al. 1993. A summary of field test methods in fractured rocks, in : Bear J., Marsily G. de, Tsang C.F. (éds), Flow and Contaminant Transport in Fractured Rock, Acddemic Press, Orlando, $560 \mathrm{p}$.
King D., Bourennane H., Isambert M. et Macaire J.-J. 1999. Relationship of the presence of a non-calcareous clay-loam horizon to DEM attributes in a gently sloping area, Geoderma, 89, 95-111

Lacombe S., Sudicky E.A., Frape S.K. et Unger A.J.A. 1995. Influence of leaky borehole on cross-formational groundwater flow and contaminant transport, Water Resour. Res., 8, 1871-1882

Ould Mohamed S., Bruand A., Bertuzzi P., Bruckler L., Guillet B. et Raison L. 1997. Estimating long-term drainage at a regional scale using a deterministic model, Soil Sci. Soc. Am. J., 61, 1473-1482

Pulido-Bosch A., Andreu I.M., Estevez A. et Ayanz |. 1998. Zonation hydrogéochimique verticale dans l'aquifère karstique du Cid (Alicante, Espagne), C. R. Acad. Sci. Paris, 326, série lla, 421-426

Schnebelen N., Ledoux E., Bruand A., Darthout R., Creuzot G., Verbeque B. et Courtemanche P. 1996. The experimental site of Villamblain : an example of an action programme to reduce the nitrogenous diffused pollution of the Beauce calcareous aquifer, in : The use of pedotransfer in soil hydrology research in Europe, Proc. II workshop, Inra, Orléans (France), 10-12/10/1996, 151-158

Xiang J. 1995. The evaluation of the flowmeter test in three-layer dquifers and the influence of dislurbed Lones, ). Hydrol., 166 (1-2), $127-145$ 